\title{
Avaliação da Assistência a Pessoas com Hipertensão Arterial em Unidades de Estratégia Saúde da Família'
}

\author{
Evaluation of Care Provided for People with Arterial \\ Hypertension in Family Health Strategy Services
}

\author{
Ernani Tiaraju de Santa Helena \\ Doutor em Medicina Preventiva. Professor Doutor do Departamento \\ de Medicina da Universidade Regional de Blumenau. \\ Endereço: Rua Antonio da Veiga, 140, Bairro Victor Konder, CEP \\ 89012-900, Blumenau, SC, Brasil. \\ E-mail: erntshळfurb.br

\section{Maria Ines Battistella Nemes} \\ Doutora em Medicina Preventiva. Professora Assistente do De- \\ partamento de Medicina Preventiva da Faculdade de Medicina \\ da USP. \\ Endereço: Av. Dr. Arnaldo, 455, 2 andar, CEP 01246-903, São \\ Paulo, SP, Brasil. \\ E-mail: mibnemesळusp.br

\section{José Eluf-Neto} \\ Doutor em Epidemiologia. Professor Titular do Departamento de \\ Medicina Preventiva da Faculdade de Medicina da USP. \\ Endereço: Av. Dr. Arnaldo, 455, 2 andar, CEP 01246-903, São \\ Paulo, SP, Brasil. \\ E-mail: jelufnetœusp.br \\ Financiamento: Edital 003/2004 FAPESC/CNPq/SUS - Processo \\ 056/922). O primeiro autor foi bolsista de doutorado do CNPq. \\ I Este estudo é parte da Tese de Doutoramento do primeiro autor \\ intitulada "Adesão ao tratamento farmacológico de pacientes \\ com hipertensão arterial em unidades de saúde da família em \\ Blumenau, SC".
}

\section{Resumo}

Objetivo: analisar a assistência a pessoas com hipertensão arterial sistêmica (HAS) prestada por equipes de Estratégia Saúde da Família (ESF) em Blumenau-SC. Métodos: foram entrevistadas 595 pessoas com HAS moradoras da área de 10 ESF. As variáveis estudadas foram: características demográficas e socioeconômicas, estilo de vida, tratamento, comorbidades, adesão ao tratamento, satisfação com o serviço e níveis pressóricos. $\mathrm{Na}$ análise, utilizaram-se os testes de "t de Student" e Qui-quadrado. Resultados: a idade média foi 6o,6 anos. Houve predomínio do sexo feminino, cor branca, casadas, com até quatro anos de estudo, sem trabalhar, das classes C e D. A média de escolaridade foi maior em pessoas brancas e das classes A e B ( $\mathrm{p}<\mathrm{o}, \mathrm{oo1}$ ). Relataram tabagismo $13,1 \%$ e consumo de álcool 23,7\%. O tempo médio de uso de medicamentos para HAS foi de 127,9 meses, em média 1,9 medicamentos, e a monoterapia com inibidores da enzima de conversão da angiotensina foi o esquema mais frequente (19,6\%). Relataram reações adversas $20,6 \%$, que estavam associadas ao maior número de medicamentos ( $\mathrm{p}<0,02)$. Mais de $90 \%$ se mostraram satisfeitos com os serviços. A prevalência de não adesão ao tratamento medicamentoso foi de 53,1\%. Apresentaram PA 140x9ommHg 69,3\%. O descontrole pressórico mostrou-se associado a não adesão, sedentarismo e classes C/D/E. Conclusões: apesar do acesso a consultas e medicamentos e da satisfação dos usuários, os valores elevados de não adesão e dos níveis pressóricos colocam como desafio a melhoria da qualidade da assistência.

Palavras-chave: Avaliação em saúde; Saúde da família; Tratamento; Hipertensão arterial. 


\section{Abstract}

Background: to analyse the health care provided for people with arterial hypertension by family health strategy teams in Blumenau, state of Santa Catarina, Brazil. Methods: overall, 595 people with arterial hypertension who live in the area of 10 family health teams were interviewed. The studied variables were: demographic and socio-economic characteristics, life style, treatment, co-morbidities, adherence to treatment, satisfaction with health service and blood pressure. Descriptive statistics and association tests (Student's t-test, ANOVA, chisquare) were used. Results: the mean age was 60.6 years old. Most are female, white, married, with four or less years of formal education, unemployed, and with low social status. White people and those with high social status had more years of schooling (p<0.001); 13.1\% reported smoking and $23.7 \%$, use of alcohol. The mean time of use of hypertension medicines was 127.9 months, on average, 1.9 medicines, and isolated ACE inhibitors were the most used medicines (19.6\%). Adverse drug reactions were reported by $20.6 \%$, which were associated with higher number of medicines ( $<<0.02)$. More than $90 \%$ were satisfied with health services. The prevalence of non-adherence to medicines was $53.1 \%$. Blood pressure $140 x 90 \mathrm{mmHg}$ was presented by $69.3 \%$ and was associated to non-adherence, sedentariness and low social status. Conclusions: although there is access to care and medicines and despite people's satisfaction with the care provided, high prevalence of non-adherence and uncontrolled blood pressure highlight the need of improving the quality of care.

Keywords: Health Evaluation; Family Health; Treatment; Arterial Hypertension.

\section{Introdução}

A hipertensão arterial sistêmica (HAS) se mantém como um dos grandes desafios da saúde pública em todo o mundo. Inquérito populacional estimou que $\mathbf{2 5}, 6 \%$ da população americana é hipertensa (definido por medidas elevadas de pressão arterial ou referir consumir medicação anti-hipertensiva), chegando a $29 \%$ entre os mais pobres e a até $39 \%$ entre mulheres negras (National Center for Health Statistics, 2005). No Brasil, estudo de revisão estimou a prevalência de HAS em $25 \%$ para a população acima de 20 anos (Passos e col., 2006).

Sabe-se, contudo, que o controle da pressão arterial não é tarefa fácil. Hyman e Pavlik (2001) afirmaram que, entre os americanos com hipertensão, $77 \%$ não possuiam controle pressórico satisfatório. Estudo populacional brasileiro sobre o manejo da HAS em comunidade constatou que $2 / 3$ se reconheciam hipertensos, $50 \%$ faziam tratamento com medicamentos e somente 1/3 tinha sua pressão arterial controlada (Piccini e Victora, 1997).

A atenção programática estruturada parece desempenhar papel importante para melhorar o controle da pressão arterial. Estudo realizado em serviço de atenção primária mostrou redução dos níveis pressóricos de pessoas mais jovens e que tinham maior frequência de comparecimento às consultas programadas (Sala e col., 1996).

Resultados desejáveis estão articulados a várias dimensões do cuidado, como o acesso aos medicamentos, à possibilidade de diálogo entre profissionais de saúde e pacientes e à maneira que estes aderem à terapêutica proposta. 0 Ministério da Saúde (Brasil, 2006a) preconiza que a atenção básica conduza atividades de promoção, prevenção, diagnóstico precoce e tratamento adequado da HAS. Recomenda que a equipe de saúde contemple os saberes de todos os profissionais envolvidos (médico, enfermeiro, auxiliar de enfermagem e agente de saúde), bem como conduza rotinas e procedimentos que ordenem as ações de saúde da equipe, em particular dos serviços organizados segundo a Estratégia de Saúde da Família (ESF).

Estudando equipes de ESF da região Sul, Piccini e colaboradores (2006) relataram que apesar de mais de $95 \%$ dos idosos com HAS utilizarem medicação 
para controlar a pressão arterial, somente a metade dos usuários conseguiu a medicação na unidade de ESF. Estudo nas regiões Sul e Nordeste mostrou que somente $46 \%$ das pessoas com HAS atendidas em unidades da ESF tiveram consultas nos últimos seis meses, taxa um pouco melhor que a observada em unidades básicas tradicionais (Fachinni e col., 2006). Sousa e colaboradores (2006) encontraram em uma unidade de ESF proporção de $60 \%$ de descontrole pressórico.

A questão da adesão ao tratamento merece particular destaque. Recente metanálise sugere que a melhora da adesão diminui a mortalidade, consultas de emergência e internações, reduz custos médicos e promove o bem-estar dos pacientes (Simpson e col., 2006). Estudos internacionais têm estimado a prevalência de adesão das pessoas com HAS entre 30 e 75\% (Marques-Contreras e col., 2002; WHO, 2003). No Brasil, o tema ainda é pouco estudado, especialmente na atenção primária. Estudos de base hospitalar encontraram frequências de não adesão variando de 10 a 77\% (Strelec e col., 2003; Muxfeld e col., 2004; Coelho e col., 2005). Na atenção primária, Prado e colaboradores (2007), em amostra de 109 pessoas atendidas em unidade de atenção primária em Florianópolis-SC, obtiveram 68\% de não adesão utilizando contagem manual de comprimidos.

Este estudo objetiva avaliar a assistência às pessoas com HAS prestada por unidades de saúde da família de Blumenau-SC.

\section{Métodos}

\section{População de estudo e amostra}

A população de estudo foi composta por pessoas com HAS moradoras das áreas de abrangência das unidades de ESF de Blumenau, Santa Catarina.

Com o objetivo de estimar a prevalência de não adesão, calculou-se o tamanho da amostra considerando uma prevalência de não adesão de 50\%, com uma precisão de $5 \%$ e intervalo de $95 \%$ de confiança.

Procedeu-se amostragem aleatória em dois estágios. No primeiro estágio, foram sorteadas 10 unidades de ESF (dentre as 34 existentes que estavam em atividade há mais de seis meses). No segundo estágio, as pessoas moradoras da área de abrangência das unidades, que eram cadastradas como hipertensas pelos agentes comunitários de saúde no Sistema de Informação da Atenção Básica (SIAB), foram selecionadas por amostragem aleatória estratificada, distribuindo o tamanho amostral proporcionalmente às pessoas com HAS cadastradas em cada unidade.

\section{Variáveis estudadas}

As variáveis de estudo relativas às pessoas foram: 1 - socioeconômicas: cor, escolaridade (categorizada em anos de estudo: o, 1 a 4, 5 a 8, 9 a 12), renda individual, ocupação, status ocupacional, classe econômica, aglomeração domiciliar; 2 - características demográficas, socioeconômicas e de estilo de vida: sexo, idade (em anos completos), estado marital, religião, consumo atual de tabaco, consumo atual de álcool, prática de atividades físicas.

A ocupação foi codificada e agrupada com base na Classificação Brasileira de Ocupações - CBO. A classe econômica foi construída com base nos Critérios de Classificação Econômica do Brasil, que tem por referência a escolaridade e os bens de consumo (ABEP, 2005).

Em relação à doença e ao tratamento da HAS foram analisados: tempo de tratamento (em meses), regime terapêutico atual (número de medicamentos, número de anti-hipertensivos e classes de medicamentos utilizados), necessidade de ajuda para tomar medicamentos, relato de reações adversas, interrupção prévia do tratamento e não adesão atual ao tratamento medicamentoso.

Os medicamentos consumidos foram classificados com base nos grupamentos propostos na Classificação ATC (Anatomical-Therapeutical-Chemical) da Organização Mundial de Saúde (WHO, 2006).

A não adesão foi medida através do Questionário de Adesão a Medicamentos - Equipe Qualiaids (QAM-Q) previamente validado que consta de três perguntas a partir das quais se construiu uma medida composta. Somente foi considerado aderente o indivíduo que relatasse ter, nos últimos sete dias, tomado de 80 a $120 \%$ das doses prescritas, de modo correto (sem "feriados", "tomada errática", "meiaadesão" ou abandono) e relatasse que sua pressão arterial estava normal na última vez que mediu (Santa Helena e col., 2008). 
Foram ainda analisados o nível pressórico atual e o relato de outras doenças. Para detecção de transtornos mentais comuns utilizou-se o Self-Reporting Questionnaire (SRQ-2o), um instrumento validado de identificação desses transtornos na atenção primária (Mari e Williams, 1986).

As dimensões individuais da assistência à saúde avaliadas foram: tipos de serviços de saúde frequentados, fonte de obtenção dos medicamentos, procura por hospital no último ano, data da última consulta, comparecimento a consultas e atividades de grupo, opinião sobre consumo de medicamentos e satisfação com o serviço de saúde.

A satisfação com o serviço de saúde foi estimada por oito questões previamente validadas (Daneluz e col., 2006) que se referem a: estrutura e acesso ao serviço (2 questões), atendimento, tratamento e cuidado do pessoal da unidade (3 questões), satisfação com tratamento e com médico (2 questões), satisfação geral (1 questão). Constituiu-se um índice de satisfação geral, que foi obtido pela composição dos escores médios das 8 questões, sendo um a máxima satisfação, e quatro totalmente insatisfeito. Após a obtenção dos escores médios, dividiu-se a amostra (pela mediana), constituindo-se o grupo dos "mais satisfeitos" e o dos "menos satisfeitos".

\section{Coleta de dados}

Os dados foram obtidos mediante entrevistas pessoais (com cerca de uma hora de duração) realizadas na residência das pessoas com HAS por entrevistadores previamente treinados. Os entrevistados foram informados do estudo por meio da leitura do Termo de Consentimento Livre e Esclarecido. Àqueles que concordaram em participar, assinando o Termo, foi aplicado um questionário que fora submetido previamente a teste-piloto.

Foram realizadas duas aferições da pressão arterial (no meio e ao final da entrevista) com esfigmomanômetro aeroide, seguindo metodologia preconizada na IV Diretrizes Brasileiras de Hipertensão Arterial, sendo considerada pressão arterial elevada medidas maiores ou iguais a $140 \mathrm{mmHg}$ de pressão sistólica média ou 9ommHg de pressão diastólica média (Mion Jr. e col., 2004).

Todos os questionários foram revisados pelos pesquisadores para controle de qualidade do pre- enchimento. Foram consultados os prontuários dos pacientes nas unidades de saúde visando esclarecer ou complementar as informações obtidas nas entrevistas. Os dados foram digitados em banco de dados eletrônico (aplicativo Epidata 3.o, domínio público), sendo os pacientes identificados somente por número de registro.

$\mathrm{O}$ estudo foi feito em conformidade com a Declaração de Helsinque e aprovado pela Comissão de Ética em Pesquisa da Universidade Regional de Blumenau, de acordo com o parecer constante do processo de número 040/2004.

\section{Análise}

Inicialmente foram examinadas as frequências simples com intervalos interquartílicos das variáveis. Paras variáveis quantitativas foram calculadas as medidas de tendência central (média, mediana e moda) e de dispersão (desvio-padrão).

As variáveis foram apresentadas nas formas tabular e gráfica e descritas por frequência absoluta, frequência relativa e respectivos intervalos de $95 \%$ de confiança.

Foram calculadas as prevalências de não adesão, níveis pressóricos elevados e reações adversas com intervalo de $95 \%$ de confiança para uma distribuição binomial. Essas variáveis, assim como a satisfação dos usuários com os serviços, foram tomadas como aproximação do resultado da assistência prestada (desfecho principal).

Para comparação entre valores médios de algumas variáveis numéricas com distribuição normal em relação a variáveis dicotômicas, utilizou-se a estatística "t de Student" com abordagem unicaudal. Para se testar a possível associação entre variáveis qualitativas, utilizou-se o teste de "Qui-quadrado de Pearson" (ou Teste Exato de Fischer, quando fosse o caso) para estabelecer significância estatística (Altman, 1991).

Foi aceito um valor de $\mathrm{p}<0,05$ para o estabelecimento de significância estatística.

\section{Resultados}

Foram estudadas 595 pessoas com HAS. A tabela 1 apresenta a distribuição das pessoas com relação a alguns caracteres sociodemográficos. 
Tabela I - Número, proporção (\%) e respectivos intervalos de $95 \%$ de confiança das pessoas com HAS segundo características sociodemográficas, atendidas em unidades da ESF, Blumenau-SC*

\begin{tabular}{|c|c|c|c|}
\hline Características & Frequência absoluta & Frequência relativa (\%) & Intervalo de $95 \%$ de Confiança \\
\hline \multicolumn{4}{|l|}{ Sexo } \\
\hline Masculino & 176 & 29,6 & $25,9-33,4$ \\
\hline Feminino & 419 & 70,4 & $66,6-74,1$ \\
\hline \multicolumn{4}{|l|}{ Faixa etária } \\
\hline Até 39 anos & 26 & 4,4 & $2,9-6,3$ \\
\hline 40 a 49 anos & 86 & 14,5 & $11,7-17,5$ \\
\hline 50 a 59 anos & 191 & 32,1 & $28,4-36,0$ \\
\hline 60 a 69 anos & 140 & 23,5 & $20,2-27,2$ \\
\hline 70 a 79 anos & 122 & 20,5 & $17,3-24,0$ \\
\hline \multicolumn{4}{|l|}{ Estado marital } \\
\hline Casado & 378 & 63,6 & $59,6-67,5$ \\
\hline Solteiro & 23 & 3,9 & $2,5-5,8$ \\
\hline Separado & 51 & 8,6 & $6,5-11,1$ \\
\hline Viúvo & 142 & 23,9 & $20,5-27,5$ \\
\hline \multicolumn{4}{|l|}{ Raça/cor } \\
\hline Branca & 480 & 80,7 & $77,3-83,8$ \\
\hline Preta/parda & 108 & 18,2 & $15,1-21,5$ \\
\hline Outra & 37 & 6,2 & $4,4-8,5$ \\
\hline \multicolumn{4}{|l|}{ Religião } \\
\hline Protestante/Evangélica & 112 & 18,9 & $15,8-22,3$ \\
\hline Católica & 461 & 77,7 & $74,2-81,0$ \\
\hline Outras & 18 & 3,0 & $1,8-4,8$ \\
\hline Sem religião & 2 & 0,3 & $0,1-1,2$ \\
\hline
\end{tabular}

* Excluídos os ignorados.

A média de idade foi 60,6 anos $(\mathrm{dp}=12,2)$, mediana de 59,6 anos. Não se observou diferença entre as médias de idade entre os sexos.

O número médio de anos de estudo foi de 3,6 anos $(\mathrm{dp}=2,7)$ e mediana de 4 anos, sendo que a maioria $(466,78,5 \%)$ tinha até 8 anos de estudo e $92(15,5 \%)$ eram analfabetos. Não houve diferença de escolaridade segundo o sexo. Contudo, pessoas brancas tiveram em média mais tempo de estudo, 3,76 anos, do que não brancas, com 3,13 anos ( $\mathrm{t}=2,29$; $\mathrm{p}<0,05)$, assim como as pessoas das classes A/B (6,o anos) comparado com aquelas que pertenciam às classes C/D/E, com 3,2 anos ( $\mathrm{t}=10,2 ; \mathrm{p}<\mathrm{O}, \mathrm{Oo} 1)$.

A maioria (449, 75,5\%) não trabalhava, sendo que 293 (49,2\%) eram aposentadas e 114 (19,2\%) estavam sem trabalho. A média de idade das pessoas que trabalhavam era menor do que a das que não trabalhavam, respectivamente, 50,4 e 63,9 anos ( $t=13,1$; $\mathrm{p}<\mathrm{O}, \mathrm{Ooo1})$. O agrupamento ocupacional mais frequente foi dos trabalhadores industriais $(134,22,5 \%)$, seguido dos prestadores de serviço (131, 22,2\%) e dos trabalhadores do comércio (126, 21,1\%).

A renda média individual foi de $\mathrm{R} \$ 501,38$ ( $d p=418,00)$, e a mediana, de R\$36o,oo. Observou-se predomínio de pessoas nas classes C (292, 49,1\%) e D (201, 33,8\%), enquanto a média de aglomeração domiciliar foi de 1,3 ( $d p=0,7)$, e mediana 1,3 pessoas por quarto do domicílio (Tabela 2). Pessoas classificadas nas classes A/B tinham renda individual média superior $(\mathrm{R} \$ 779,80)$ comparada à das pessoas das classes $\mathrm{C} / \mathrm{D} / \mathrm{E}$ que tinham renda média de $\mathrm{R} \$ 452,90$ $(t=6,8 ; p<0,001)$. 
Tabela 2 - Número, proporção (\%) e respectivos intervalos de $95 \%$ de confiança das pessoas com HAS segundo características socioeconômicas, atendidas em unidades da ESF, Blumenau-SC*

\begin{tabular}{|c|c|c|c|}
\hline Características & Frequência absoluta & Frequência relativa (\%) & Intervalo de $95 \%$ de Confiança \\
\hline \multicolumn{4}{|l|}{ Escolaridade } \\
\hline Analfabetos & 92 & 15,5 & $12,7-18,7$ \\
\hline I a 4 anos & 382 & 64,3 & $60,3-68,2$ \\
\hline 5 a 9 anos & 84 & 14,1 & $11,4-17,2$ \\
\hline Médio/Superior & 36 & 6,1 & $4,3-8,3$ \\
\hline \multicolumn{4}{|l|}{ Grupo Ocupacional } \\
\hline Chefias/Comércio/Serviços & 321 & 54,0 & $49,9-58,0$ \\
\hline Agricultores & 53 & 8,9 & $6,7-11,5$ \\
\hline Industriários & 134 & 22,5 & $19,2-26,1$ \\
\hline Trab. braçais não qualificados & 87 & 14,6 & $11,9-17,7$ \\
\hline \multicolumn{4}{|l|}{ Status ocupacional } \\
\hline Empregado & 101 & 17,0 & $14,0-20,2$ \\
\hline Empregador & 4 & 0,7 & $0,2-1,7$ \\
\hline Autônomo & 41 & 6,9 & $5,0-9,2$ \\
\hline Auxílio doença & 42 & 7,1 & $5,1-9,4$ \\
\hline Aposentado & 293 & 49,2 & $45,2-53,3$ \\
\hline Sem trabalho & 114 & 19,1 & $16,1-22,6$ \\
\hline \multicolumn{4}{|l|}{ Renda individual } \\
\hline Mais de I salário mínimo & 275 & 50,4 & $46,1-54,6$ \\
\hline Até I salário mínimo & 271 & 49,6 & $45,4-54,0$ \\
\hline \multicolumn{4}{|l|}{ Classes econômicas } \\
\hline A & 1 & 0,2 & $0,0-0,9$ \\
\hline B & 92 & 15,4 & $12,7-18,6$ \\
\hline c & 292 & 49,1 & $45,0-53,2$ \\
\hline D & 201 & 33,8 & $30,0-37,7$ \\
\hline E & 9 & 1,5 & $0,7-2,9$ \\
\hline \multicolumn{4}{|l|}{ Aglomeração domiciliar } \\
\hline Até I pessoa/quarto & 283 & 48,5 & $44,3-52,5$ \\
\hline Mais que I pessoa/quarto & 301 & 51,5 & $47,4-55,7$ \\
\hline
\end{tabular}

* Excluidos os ignorados.

O número de tabagistas foi de 78 (13,1\%, IC $95 \%$ 10,5 - 16,1) e $141(23,7 \%$, IC95\% 20,3 - 27,3) relataram consumo de álcool. Encontrou-se uma maior proporção de tabagistas entre aqueles que consumiam álcool (35,9\% vs 21,9\%, 2=7,39, $\mathrm{p}<0,01)$. A maioria das pessoas $(423,71,1 \%$, IC $95 \% 67,3-74,7)$ não praticava atividade física (fora o trabalho) nos últimos sete dias. As 172 pessoas que relataram praticar atividade física o faziam em média 3,6 dias $(\mathrm{dp}=2,2)$ e mediana de 3 dias. Não se observou associação entre tabagis- mo ou consumo de álcool e não praticar atividade física. O hábito de fumar e o consumo de álcool se mostraram inversamente associados com idade (respectivamente $\mathrm{t}=3,24 ; \mathrm{p}<0,01$ e $\mathrm{t}=4,9$; $\mathrm{p}<0,0001$ ).

A média de tempo de doença foi de 141,2 meses $(\mathrm{dp}=113,7)$, e mediana de 120 meses. Dentre as doenças associadas referidas, observou-se que 267 (44,8\%, IC95\% 40,8-49,o) pessoas com HAS tinham diabetes mellitus e 147 (24,7\%, IC95\% 21,3 - 28,4) tinham algum tipo de dislipidemia. 
Destaca-se a presença de transtornos mentais comuns em 265 dos entrevistados (44,8\%, IC $95 \%$ 40,5 - 48,6), os quais apresentavam tempo médio desde a última consulta menor que os demais $(75,2$ dias e 110,6 dias, respectivamente - $\mathrm{t}=3,7 ; \mathrm{p}<\mathrm{o}, \mathrm{oo1}$ ).

Relataram ter interrompido previamente o tratamento sem autorização do médico 169 pessoas, sendo que 72 (12,2\%, IC95\% 9,7 - 15,1) interromperam 1 vez, 53 (9,0\%, IC95\% 6,8 - 11,6) interromperam de 2 a 4 vezes e 44 pessoas $(7,5 \%$, IC $95 \% 5,5-9,9)$ interromperam 5 ou mais vezes. Interrupção prévia mostrou-se associada à média de idade mais baixa (56,1 vs. 62,3 anos, $\mathrm{t}=5,7 \mathrm{p}<0,0001)$ e com relato de reações adversas ( $37,1 \%$ vs. $24,2 \%, 2=7,75$ p<0,o1).
A tabela 3 apresenta variáveis relativas aos serviços de saúde. A média de tempo decorrido desde a última consulta foi de 94 dias ( $d p=116,4)$, e mediana de 6 o dias. Importante frisar que $25 \%$ das pessoas se consultaram nos últimos 28 dias e $75 \%$, nos últimos 120 dias. Pessoas inseridas no mercado de trabalho apresentaram maior tempo desde a última consulta quando comparado àquelas que não trabalhavam, respectivamente, 121,6 e 85,5 dias ( $\mathrm{t}=3,2 ; \mathrm{p}<\mathrm{o}, \mathrm{o} 1)$.

Com relação ao consumo de medicamentos, 24 $(4,0 \%$ IC $95 \% 2,6-5,9)$ das 595 pessoas entrevistadas não faziam uso de qualquer tipo de medicamento. Dentre as demais, a média foi de 4,6 medicamentos $(\mathrm{dp}=2,4)$, e a mediana de 4 medicamentos.

Tabela 3 - Número, proporção (\%) e respectivos intervalos de $95 \%$ de confiança das pessoas com HAS segundo características relativas à assistência prestada por serviços de saúde, atendidas em unidades da ESF, Blumenau-SC*

\begin{tabular}{lccc} 
Relação com serviços de saúde & Frequência absoluta & Frequência relativa (\%) & Intervalo de 95\% de Confiança \\
Tipo de serviço médico & 104 & 17,5 & $14,5-20,8$ \\
Particular/Convênio & 491 & 82,5 & $79,2-85,5$ \\
SUS & & & \\
Fonte dos medicamentos & 575 & 96,6 & $94,9-97,9$ \\
SUS & 20 & 3,4 & $2,1-5,1$ \\
Precisou comprar & & & \\
Comparecimento às consultas & 519 & 89,6 & $86,9-92,0$ \\
Sim & 60 & 10,4 & $8,0-13,1$ \\
Não & & & \\
Comparecimento aos grupos & 147 & 25,0 & $21,5-28,7$ \\
Sim & 442 & 75,0 & $71,3-78,4$ \\
Não & & & $78,7-85,0$ \\
Tempo da última consulta & 483 & 82,4 & $14,5-20,8$ \\
Até 6 meses & 103 & 17,6 & \\
6 meses e mais & & & $27,7-35,3$ \\
Procurou hospital nos últimos 12 meses & 187 & 31,4 & $64,7-72,3$ \\
Sim & 408 & 68,6 & \\
Não & & & \\
\hline
\end{tabular}

*Excluídos os ignorados.

Faziam uso regular de medicamentos anti-hipertensivos 565 pessoas, com média de $1,9(\mathrm{dp}=0,9)$ e mediana de 2 medicamentos. Detectou-se 30 casos de abandono total do tratamento. As classes de medicamentos anti-hipertensivos mais utilizadas foram os inibidores da enzima de conversão da angiotensina - IECA (373, 34,8\%, IC95\% 31,9-37,7), seguidos dos diuréticos tiazídicos (279, 26,o\%, IC $95 \%$ 23,4 - 28,7) e beta-bloqueadores (220, 20,5\%, IC $95 \%$ 18,1 - 23,0). 0 esquema terapêutico mais utilizado foi a monoterapia com IECA para 111 pessoas (19,6\%, IC95\% 16,5 - 23,2), seguido da associação de diuréticos tiazídicos e IECA para 105 pessoas $(18,6 \%$, IC $95 \%$ 15,2 - 22,o). A média de tempo de tratamento 
com anti-hipertensivos foi de 127,9 meses ( $d p=106,3)$, e a mediana de 96 meses.

Somente 73 pessoas $(12,8 \%$, IC95\% 10,2 - 15,9) relataram necessitar de algum tipo de ajuda para tomar seus medicamentos, com maior proporção de pessoas solteiras, separadas ou viúvas $(18,5 \%$ vs. $9,5 \%, \quad 2=9,51 \mathrm{p}<0,01)$.

Através do uso do QAM-Q, 316 pessoas foram consideradas não aderentes, representando uma prevalência de não adesão de 53,1\% (IC 95\% 49,o57,2).

A medida de pressão arterial mostrou que 404 pessoas $(69,3 \%$, IC95\% 65,4 - 73,0) apresentaram níveis pressóricos sistólicos maiores ou iguais a $140 \mathrm{mmHg}$ ou diastólicos maiores ou iguais a 9ommHg.

A maior proporção de níveis pressóricos elevados se mostrou associada a algumas características das pessoas, como: cor preta-parda $(79,8 \%$ vs. $66,7 \%$, $2=7,38 \mathrm{p}<0,01)$, ter outras doenças associadas $(75,5 \%$ vs. $65,7 \%, 2=6,18, p<0,05)$, não praticar atividade física $(71,9 \%$ vs. $62,9,2=4,56, \mathrm{p}<0,05)$, ser das classes econômicas C/D/E (71,0\% vs. 6o,2\%, 2=4,29, p<0,05), além de variáveis do serviço como ser atendido pelos médicos do SUS, sejam da ESF ou especialistas, ( $71,6 \%$ vs. 58,o, 2=7,24, p<0,o1) e a não adesão aos medicamentos (77,8\% vs. 59,6\%, 2=22,71, $\mathrm{p}<0, \mathrm{ooo1})$.

Após iniciar o uso dos anti-hipertensivos, 117 pessoas (20,6\%, IC 95\% 17,3-24,1) relataram sentir algum tipo de reação adversa. As que relataram reações adversas tomavam, em média, mais medicamentos do que aquelas que não relataram (5,2 e 4,4 , respectivamente $(\mathrm{t}=3, \mathrm{O} 7, \mathrm{p}<\mathrm{O}, \mathrm{O} 1)$ ), e as reações eram mais frequentes entre aquelas que tomavam 3 ou mais anti-hipertensivos ( $24,4 \%$ vs. $14,9 \%, \chi 2=8,19$ $\mathrm{p}<\mathrm{O}, \mathrm{O} 1)$.

A maioria (36o, 64,3\%, IC95\% 6o,2 - 68,3) considerou adequada a quantidade de medicamentos que usa. O grau de satisfação do usuário com o serviço de saúde foi medido por questionário com oito questões. 0 gráfico 1 mostra a proporção de satisfação por item do questionário.

\section{Gráfico I - Proporção (\%) das pessoas com HAS segundo grau de satisfação com o serviço de saúde, atendidas em unidades da ESF, Blumenau-SC*}

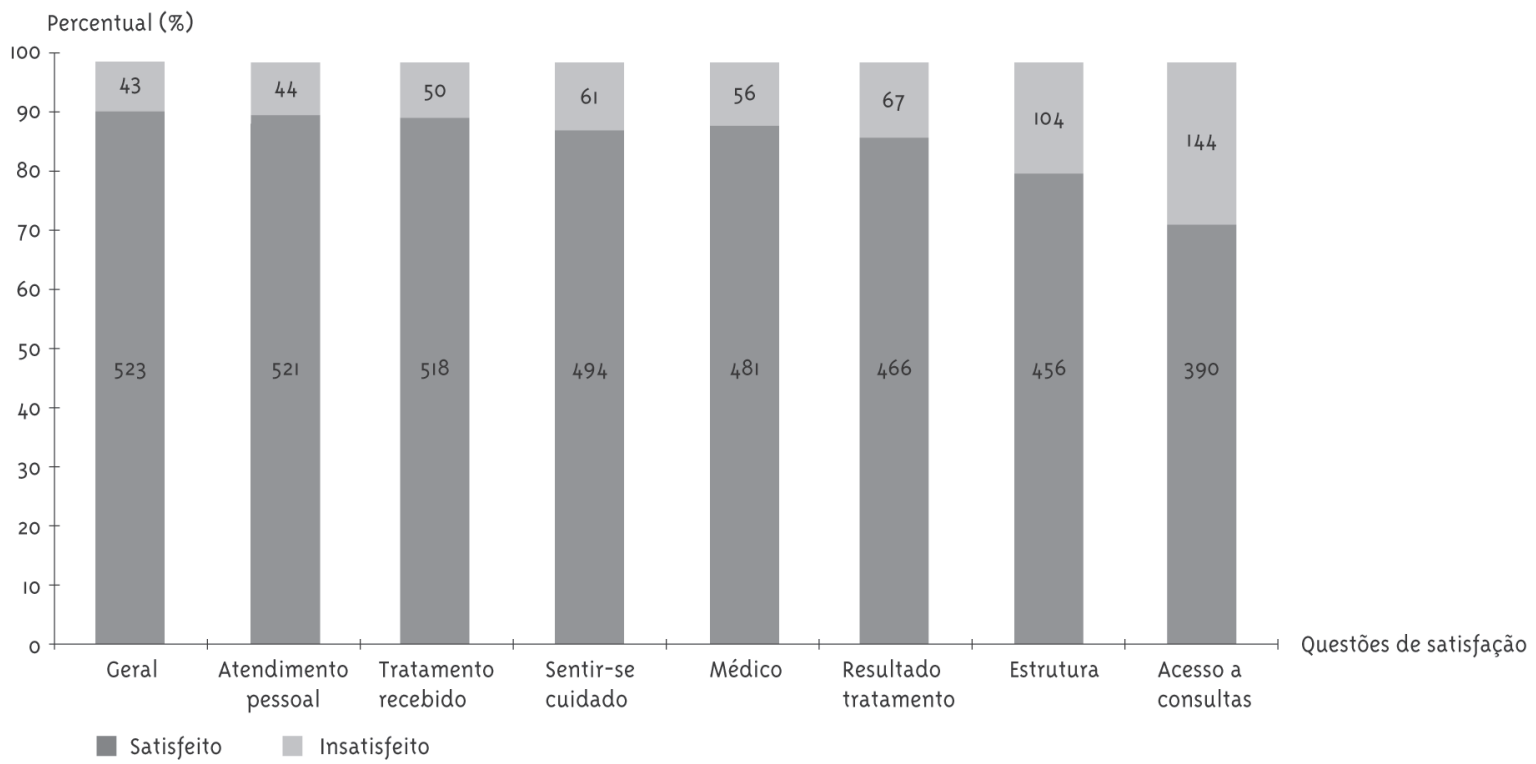


Os usuários se mostraram mais satisfeitos com aspectos relacionais, como: $92,2 \%$ estão satisfeitos com o atendimento e acham-no muito bom. No entanto, nos itens de estrutura da unidade e acesso a consultas, a satisfação cai para 81,2 e 73\%, respectivamente. Maior proporção de pessoas satisfeitas com os serviços se mostrou associada com ausência de transtornos mentais comuns $(63,6 \%$ vs. $42,9 \%$, $2=24,32$ p<0,0001), adesão aos medicamentos (50,5\% vs. $41,3 \%, 2=4,75 \mathrm{p}<0,05)$ e ausência de reações adversas $(82,7 \%$ vs. $75,2 \%, 2=4,57 \mathrm{p}<0,05)$. Contudo, não se observou associação entre opinião sobre o uso de medicamentos e satisfação com serviços e os resultados desejáveis do tratamento (controle de nível pressórico).

\section{Discussão}

O perfil sociodemográfico das pessoas com HAS participantes deste estudo se mostrou semelhante ao perfil das pessoas com HAS cadastradas no sistema de informações sobre HAS/Diabetes das unidades básicas de saúde (Hiperdia). Corresponde também ao perfil predominante de pessoas cobertas pelas equipes de ESF do município de Blumenau, quanto ao sexo, idade e escolaridade (Brasil, 20o6b).

Dentre os pressupostos para implantação de unidades de ESF destaca-se a proposta de melhorar o acesso de pessoas socialmente desfavorecidas, isto é, mediante o acesso aos cuidados de saúde melhorar a equidade (Brasil, 2002). O perfil observado e a associação entre as características socioeconômicas (pessoas de baixa escolaridade, cor não branca, baixa renda, baixo poder de consumo e aposentados) permitem supor que os participantes deste estudo, em sua maioria, são pessoas em posição socialmente desfavorável.

A proporção de fumantes atuais foi menor que a descrita em estudos populacionais (Costa e col, 2007). Inquérito de saúde populacional realizado nas principais capitais mostrou uma frequência que variou entre 14,1 e $25,6 \%$ em pessoas com 25 anos (Brasil, 2004). Os resultados obtidos neste estudo podem representar problemas de acurácia no instrumento utilizado ou uma maior adesão das pessoas às recomendações dos profissionais de saúde para assumirem hábitos de vida mais saudáveis.
Houve um predomínio de diabetes mellitus associada com HAS (44,8\%), valor elevado se comparado a outros estudos, como Muxfeld e colaboradores (2004) com 29,8\%. Porém, a associação com dislipidemias em $24,7 \%$ dos casos se mostrou inferior ao descrito por esses autores (44\% e 38\%, respectivamente). Essas variações podem refletir características próprias da população estudada ou ainda ser resultado de possível viés de seleção amostral.

A prevalência de transtornos mentais comuns se mostrou elevada se comparada a estudos de base populacional em diversas cidades brasileiras, que encontraram valores entre 24 e 35\% (Maragno e col., 20o6; Ludemir e Melo Filho, 2002). Contudo, outros estudos realizados em populações atendidas em serviços de atenção primária obtiveram valores mais elevados (37 a 51\%), dependendo do método utilizado (Gonçalves e Kapczinski, 20o8; Bandeira e col., 2007; Fortes e col., 2008). Essa prevalência mais elevada pode ser explicada, ao menos em parte, por se tratar de pessoas em condições de maior vulnerabilidade social (Lima e col., 1999).

A baixa proporção de faltosos à consulta nos últimos seis meses, em oposição ao número de faltosos às atividades de grupos, sugere que a atenção individual é mais valorizada pelos pacientes. Merece destaque que mais $50 \%$ das pessoas se consultaram nos últimos 60 dias, e que $96 \%$ obtiveram seus medicamentos no SUS, o que sugere facilidade de acesso aos serviços e insumos. Estudo de avaliação da ESF no município de São Paulo mostrou que o acesso foi uma das dimensões melhores avaliadas pelos usuários da ESF (Elias e col., 2006). Além de ter acesso, comparecer a consultas também parece ser importante para melhoria do controle clínico em pessoas com HAS (Coelho e col., 2005; Sala e col., 1996).

Os resultados parecem melhores que os apresentados por outros estudos de avaliação da ESF. Estudo sobre atenção à saúde dos idosos apontou que, na região Sul, 50\% dos idosos com HAS se consultaram na unidade de ESF, 53\% obtiveram medicamentos na unidade e 31\% compareceram aos grupos (Piccini e col., 2006). Outro estudo relata que, na região Sul, a proporção de adultos hipertensos que se consultaram nos últimos seis meses nas unidades de ESF $(46,5 \%)$ foi superior aos $31,4 \%$ atendidos em unida- 
des básicas tradicionais (Fachinni e col., 2006).

0 esquema terapêutico predominante para HAS estava em consonância ao recomendado nas IV Diretrizes para tratamento da HAS. Outros estudos, em contexto de atenção primária, também identificaram esquemas semelhantes, ao passo que estudos em serviços especializados tiveram predomínio de esquemas com dois ou mais medicamentos (Strelec e col., 2003; Muxfeld e col., 2004), o que pode ser explicado, ao menos em parte, pelo fato de serviços especializados ou de referência assistirem pessoas com quadro clínico refratário e/ou de maior complexidade.

A adesão ao tratamento reflete, ao menos em parte, o modo como as pessoas compreendem e assumem o cuidado com sua saúde. Nesse sentido, contém um forte componente relacional manifesto nos processos interativos entre pessoas que necessitam de cuidado e o serviço de saúde que deve prestá-lo. Assim, é licito tomar a adesão à terapêutica como parte do resultado desejável do cuidado em saúde e, por conseguinte, tomar a prevalência de não adesão como indicador de problemas na qualidade do processo de cuidado em saúde.

A prevalência de não adesão observada foi maior do que a média obtida, de 39,6\%, em revisão de estudos na atenção primária da Espanha (Marquez-Contreras e col., 2002) e menor do que a obtida por Prado e colaboradores (2007), de 68, $2 \%$, em estudo em um serviço de atenção primária em Florianópolis-SC.

Níveis pressóricos elevados se mostraram associados a condições sociais desfavoráveis, bem como a atendimento por médico do SUS e não aderir ao tratamento, o que indica falhas na qualidade da atenção prestada, em particular aos indivíduos de pior condição social, justamente os que deveriam receber cuidado diferenciado, conforme tem sido preconizado nas Diretrizes Nacionais de HAS e no Programa Nacional de Hipertensão Arterial do Ministério da Saúde (Mion Jr. e col., 2004; Brasil, 2006b).

A associação observada entre polifarmácia com anti-hipertensivos e uso excessivo de outros medicamentos com reações adversas se mostra consistente com outros estudos (Malhotra e col., 2001; Martinez Vergara e col., 2005). Parte dessas reações adversas pode resultar de interações medicamentosas, problema crescente entre pessoas idosas que usam muitos medicamentos. O uso de monoterapia, baixas doses e maior individualização do tratamento podem contribuir para redução das reações adversas a medicamentos e melhorar o controle clínico (Cohen, 2001).

Segundo Ware (1983), a medida da satisfação dos usuários ou dos pacientes é uma avaliação pessoal dos cuidados e dos serviços de saúde que são a eles dispensados. Assim, a satisfação pode ser influenciada pelo status emocional da pessoa no seu momento atual.

Neste estudo, as frequências observadas dos itens de satisfação sugerem que a maioria dos usuários estava satisfeita com as unidades de ESF, com melhor desempenho para questões relacionais. Os itens que apresentaram pior desempenho se referiam à estrutura e ao acesso. Diante da associação observada entre satisfação e transtornos mentais comuns, parece recomendável relativizar tais resultados. No entanto, esses resultados se mostraram semelhantes aos encontrados por Trad e colaboradores (2002). Os autores utilizaram metodologia qualitativa e avaliaram a satisfação dos usuários do ESF em alguns municípios do estado da Bahia, tendo encontrado melhor desempenho para as dimensões relacionais do que para as organizacionais.

Avaliações recentes sobre a Estratégia Saúde da Família sugerem que a estratégia tem melhorado o acesso a consultas, exames e medicamentos, além de praticar a territorialização, o vínculo e a atenção programática comparados com unidades de saúde tradicionais (Fachinni e col., 2006; Elias e col., 2006; Conill, 2008).

Os indicadores de monitoramento do Pacto de Atenção Básica em Santa Catarina e em Blumenau, em particular, mostraram um aumento no número de pessoas com HAS cadastradas (ainda que muito aquém do esperado), o que parece indicar a ampliação do acesso ao tratamento para um número maior de pessoas (Brasil, 2007).

Apesar disso, estudos em unidades de atenção primária sugerem que a melhoria dos níveis pressóricos pode ser obtida com cuidado intensivo (baixo intervalo entre consultas, manejo medicamentoso, busca ativa de faltosos, atividades educativas individuais e em grupo) e equipe multiprofissional, isto é, pelo modo como o serviço organiza seu processo 
de trabalho para dar conta de um problema de saúde (Silva e col., 2006; Didier e col., 2007).

Obter esses resultados satisfatórios não somente em uma unidade, mas no conjunto da rede de atenção primária, permanece como desafio ao gestor municipal. A este cabe garantir a estrutura necessária, bem como trabalhar pela melhoria do processo de trabalho dos profissionais de saúde da rede através de qualificação profissional, planejamento e monitoramento de ações assistenciais desenvolvidas no nível local.

Este estudo tem pelo menos três limitações que merecem destaque. Primeira: o processo amostral pode ter sofrido viés de seleção, pois as pessoas com HAS foram identificadas a partir do cadastro elaborado pelos agentes comunitários de saúde das unidades amostradas. Algumas pessoas com hipertensão podem não ter sido cadastradas (em especial quadros limítrofes ou os pacientes que não se reconhecem como hipertensos, bem como pessoas ativas no mercado de trabalho). Contudo, é lícito supor que, caso tenha ocorrido de fato esse viés, foi no sentido de diminuir a associação encontrada.

Segunda: considera-se classicamente que o uso de questionários subestima a não adesão. Uma alternativa é aumentar o ponto de corte de não adesão como forma de melhorar a sensibilidade. 0 uso da medida combinada do QAM-Q aumenta a sensibilidade por considerar aderente somente quem tomou seus medicamentos na quantidade, de modo correto e com relato de efeito favorável.

A terceira possível limitação se refere a considerar o controle clínico a partir de duas medidas de pressão arterial numa mesma entrevista. Sabe-se que a entrevista domiciliar pode representar um fator estressor ao entrevistado com possível efeito de aumento da pressão arterial. Contudo, por questões financeiras e operacionais, não foi possível realizar medidas de pressão arterial em momentos diversos.

\section{Conclusões}

A atenção a pessoas com HAS prestada nas unidades de ESF de Blumenau parece seguir a chamada "regra das metades", comumente citada na literatura médica: metade das pessoas toma seus remédios e, destas, somente a metade tem sua pressão arterial controlada.
Apesar do acesso a consultas e medicamentos e de se mostrarem satisfeitas, a baixa proporção de pessoas com pressão arterial controlada sugere que o cuidado adequado às pessoas com HAS nas unidades de ESF permanece como desafio.

\section{Referências}

Associação Brasileira de Empresas de Pesquisa ABEP. Critério de Classificação Econômica Brasil. Disponível em: <http://publicacoes.cardiol.br/ consenso/2004/Diretriz\%2oHA.pdf >. Acesso em: 20 nov. 2005.

ALTMAN, R. Practical Statistics for Medical Research. London: Chapman \& Hall, 1991.

BANDEIRA, M. et al. Avaliação da ocorrência de transtornos mentais comuns em usuários do Programa de Saúde da Família. Jornal Brasileiro de Psiquiatria, Rio de Janeiro, v. 56, n. 1, 2007, p. 41-47.

BRASIL. Ministério da Saúde. Avaliação da implementação do Programa Saúde da Família em dez grandes centros urbanos: síntese dos principais resultados. Brasília: Ministério da Saúde, 2002.

BRASIL. Ministério da Saúde. Inquérito domiciliar sobre comportamentos de risco e morbidade referida de doenças e agravos não transmissíveis: Brasil, 15 capitais e Distrito Federal, 2002-2003. Rio de Janeiro: INCA, 2004.

BRASIL. Ministério da Saúde. Secretaria de Atenção à Saúde. Departamento de Atenção Básica. Hipertensão arterial sistêmica para o Sistema Único de Saúde. Brasília: Ministério da Saúde, 2006. 58 p. (Cadernos de Atenção Básica, 16).

BRASIL. Ministério da Saúde. Atenção básica e Saúde da Família: números. Disponível em: <http://nutricao.saude.gov.br/dab/abnumeros. php>. Acesso em: 04 jun. 2006.

BRASIL. Ministério da Saúde. DATASUS. Informações de saúde. Disponível em: <http:// tabnet.datasus.gov.br/cgi/deftohtm.exe?sih/cnv/ mrsc.def >. Acesso em: 05 jun. 2007. 
COELHO, E. B. et al. Relação entre a assiduidade às consultas ambulatoriais e o controle da pressão arterial em pacientes hipertensos. Arquivos Brasileiros de Cardiologia, Rio de Janeiro, v. 85, n. 3, p. 157-161, set. 2005 .

COHEN, J. Adverse drug effects, compliance and initial doses of antihypertensive drugs recommended by National Joint Committee vs the physician's desk reference. Archives of Internal Medicine, Chicago, v. 161, p.880-885, mar. 2001.

CONILL, E. M. Ensaio histórico-conceitual sobre a atenção primária à saúde: desafios para a organização de serviços básicos e da Estratégia Saúde da Família em centros urbanos no Brasil. Cadernos de Saúde Pública, Rio de Janeiro, v. 24, Supl. 1, p. s7-s16, 2008.

DANELUZ, M. et al. Desenvolvimento e validação de questionário de satisfação dos usuários hipertensos do Programa de Saúde da Família, Blumenau, SC. In: CONGRESSO BRASILEIRO DE SAÚDE COLETIVA, 8. CONGRESSO MUNDIAL DE SAÚDE COLETIVA, o9. 2006, Rio de Janeiro. Anais... Rio de Janeiro: Associação Brasileira de Pós-Graduação em Saúde Coletiva, 2006. 1 CDRom.

DIDIER, M. T.; GUIMARÃES, A. C. Otimização de recursos no cuidado primário da hipertensão arterial. Arquivos Brasileiros de Cardiologia, Rio de Janeiro, v. 88, n. 2, p. 218-224, 2007.

ELIAS, P. E. et al. Atenção básica em saúde: comparação entre PSF e UBS por estrato de exclusão social no município de São Paulo. Ciência e Saúde Coletiva, Rio de Janeiro, v. 11, n. 3, p. 633641, 2006.

FACCHINI, L. A. et al. Desempenho do PSF no sul e no nordeste: avaliação institucional e epidemiológica da atenção básica à saúde, Ciência e Saúde Coletiva, Rio de Janeiro, v.11, n. 3, p. 669681, 2006.

FORTES, S. et al. Nosological profile and prevalence of common mental disorders of patients seen at Family Health Program (FHP) units in Petrópolis. Rio de Janeiro, Revista Brasileira de Psiquiatria, São Paulo, v. 30, n. 1, p. 32-37, mar. 2008.
GONÇALVES, D. M.; KAPCZINSKI, F. Transtornos mentais em comunidade atendida pelo Programa Saúde da Família. Cadernos de Saúde Pública, Rio de Janeiro, v. 24, n. 9, p. 2043-2053, set. 2008.

HYMAN, D. J.; PAVLIK, V. N. Characteristics of patients with uncontrolled hypertension in the United States. New England Journal of Medicine, Waltham, MA, v. 345, p. 479-86, 16 aug. 2001.

LIMA, M. S. et al. Saúde e doença mental em Pelotas, RS: dados de um estudo populacional. Revista de Psiquiatria Clínica, São Paulo, v. 26, n. 5, set./out. 1999. Disponível em: < http://www. hcnet.usp.br/ipq/revista/vol26/n5/artigo(225).htm >. Acesso em: 04 out. 2008.

LUDEMIR, A. B.; MELO FILHO, D. A. Condições de vida e estrutura ocupacional associadas a transtornos mentais comuns. Revista de Saúde Pública, São Paulo, v. 36, n. 2, p. 213-221, abr. 2002.

MALHOTRA, S. et al. Drug related medical emergencies in the elderly: role of adverse drug reactions and non-compliance. Postgraduate Medical Journal, London, v. 77, n. 913 p. 703-7, nov. 2001.

MARAGNO, L. et al. Prevalência de transtornos mentais comuns em populações atendidas pelo Programa Saúde da Família (QUALIS) no Município de São Paulo, Brasil. Cadernos de Saúde Pública, Rio de Janeiro, v. 22, n. 8, p. 16391648, ago. 2006.

MARI, J. J.; WILLIAMS, P. A validity study of a psychiatric screening questionnaire (SRQ-20) in primary care in the city of Sao Paulo. British Journal of Psychiatry, London, v. 148, p. 23-26, 1986.

MARQUEZ-CONTRERAS, E. et al. El incumplimiento terapéutico en el tratamiento de la hipertensión arterial en España. Análisis de los estudios publicados entre 1984 y 2001. Hipertensión, Madrid, v. 19, n. 1, p. 12-16, 2002.

MARTINEZ VERGARA, M. A. et al. Interacciones farmacológicas potenciales entre antihipertensivos y otros medicamentos de uso crônico. Hipertensión, Madrid, v. 22, n. 24, p. 146 50, 2005. 
MION JR, D. et al. IV Diretrizes Brasileiras de Hipertensão Arterial. Arquivos Brasileiros de Cardiologia, São Paulo, v. 82, p.1-14, 2004. Suplemento 4 .

MUXFELD, E. S. et al. Demographic and clinical characteristics of hypertensive patients in the internal medicine outpatient clinic of a university hospital in Rio de Janeiro. Sao Paulo Medical Journal, São Paulo, v. 122, n. 3, p. 87-93, may 2004. NATIONAL CENTER FOR HEALTH STATISTICS. Health, United States, 2005: with chartbook on Trends in the Health of Americans. Hyattsville, Maryland: 2005

PASSOS, V. M. A. et al. Hipertensão arterial no Brasil: estimativa de prevalência a partir de estudos de base populacional. Epidemiologia e Serviços de Saúde, Brasília, v. 15, n. 1, p. 35-45, jan.-mar. 2006.

PICCINI, R. X. et al. Necessidades de saúde comuns aos idosos: efetividade na oferta e utilização em atenção básica à saúde. Ciência e Saúde Coletiva, Rio de Janeiro, v. 11, n. 3, p. 657$667,2006$.

PICCINI, R. X.; VICTORA, C. G. How well is hypertension managed in the community? A population-based survey in a Brazilian city. Cadernos de Saúde Pública, Rio de Janeiro, v. 13, n. 4, p. 595-6oo, out./dez. 1997.

PRADO, J. C. et al. Validity of four indirect methods to measure adherence in primary care hypertensives. Journal of Human Hypertension, London, v. 21, n. 7, p. 579-84, july 2007.

SALA, A. et al. Avaliação da efetividade do controle da hipertensão arterial em unidade básica de saúde. Revista de Saúde Pública, São Paulo, v. 3o, n. 2, p. 161-7, abr. 1996.

SANTA HELENA, E. T.; ELUF-NETO, J.; NEMES, M. I. B. Desenvolvimento e validação de questionário para medir não-adesão ao tratamento com medicamentos. Revista de Saúde Pública, São Paulo, v. 42, n. 4, p.764-767, ago. 2008.
SILVA, T. R. et al. Controle de Diabetes Mellitus e Hipertensão Arterial com grupos de intervenção educacional e terapêutica em seguimento ambulatorial de uma unidade básica de saúde. Saúde e Sociedade, São Paulo, v. 15, n. 3, p. 180189, set./dez. 2006.

SIMPSON, S. H. et al. A meta-analysis of the association between adherence to drug therapy and mortality. British Medical Journal, London, v. 333, n. 7557, p.15-19, 21 o1 july 2006.

SOUSA, L. B. et al. Hipertensão arterial e saúde da família: atenção aos portadores em município de pequeno porte na Região Sul do Brasil. Arquivos Brasileiros de Cardiologia, Rio de Janeiro, v. 87, p. 496-503, oct. 2006.

STRELEC, M. A. A. M. et al. The influence of patient's consciousness regarding high blood pressure and patient's attitude in face of disease controlling medicine intake. Arquivos Brasileiros de Cardiologia, Rio de Janeiro, v. 81, n. 4, p. 349-54, oct. 2003 .

TRAD, L. et al. Estudo etnográfico da satisfação do usuário do Programa de Saúde da Família (PSF) na Bahia. Ciência e Saúde Coletiva, Rio de Janeiro, v. 7, n. 3, p. 581-589, 2002.

WARE, J. E. Defining and measuring patient satisfaction with medical care. Evaluation of Program and Plannig, Amsterdam, v. 6, n. 3/4, p. 247-263, 1983.

WHO - Collaborating Centre for Drug Statistics Methodology Norwegian Institute of Public Health Adherence to long-term therapies: evidence for action. Geneve: WHO; 2003.

WHO - Collaborating Centre for Drug Statistics Methodology Norwegian Institute of Public Health. Anatomical Therapeutic Chemical (ATC) Classification. Disponível em: <http://www.whocc. no/atcddd/>. Acesso em: 04 jun. 2006.

Recebido em: 10/02/2009

Reapresentado em: 03/07/2009

Aprovado em: 05/09/2009 